\title{
Gravity until equipartition of relativistic kinetic energies
}

\author{
Igor E. Bulyzhenkov ${ }^{1,2, a}$ \\ ${ }^{1}$ Moscow Institute of Physics and Technology, 9 Institutskiy per., Dolgoprudny, Moscow reg., 141700, Russia \\ ${ }^{2}$ Lebedev Physics Institute RAS, 53 Lininsky pros., Moscow, 119991, Russia
}

\begin{abstract}
Einstein's relativistic mechanics can describe the low speed transport of variable internal and translation energies through two independent degrees of freedom while Newton's model of cold masses always operates only with one degenerated degree for these kinetic energies. Despite similar mathematics with Newton, Einstein's low speed physics for the spatial transport of variable internal heat and kinetic energy of ordered translations differs essentially from the classical physics of constant masses. Gravity of mechanical bodies with internal relativistic heat originates from the universal tendency of kinematic energies to their dynamical equipartition over available degrees of freedom. The free fall deceleration in extreme fields is followed by the accelerated takeoff and by further oscillations around the kinematic energy equilibrium.
\end{abstract}

\section{Introduction}

Since the high school we all are getting used to the wrong opinion that Einstein's relativistic physics in weak fields should reproduce Newtonian physics (but not only mathematics) for the slow motion, $v^{2} \ll c^{2}$, of constant point masses. Despite the fact that the classical mechanics of such masses does not know numerical failures "in the field of its applicability", the Newtonian ontology can not be correct if we accepted another low speed mechanics with extra (internal) degrees of energy freedom. Contrary to the extended Cartesian vortex or the Einstein particle with internal relativistic heat, the Newtonian probe body has no internal energy contents. But different degrees of freedom divorce Newton physics of constant masses and Einstein physics of variable energies in principle. The goals of this note is to criticize physics of the point mass, to clarify quantitatively why "Einstein's theory can be accepted only with the recognition that Newton's was wrong" [1], and to propose the kinematic reason for the origin of gravity.

The inertial or gravitational charge in Einstein's physics is proportional to the full energy, $E_{o} \sqrt{g_{o o}} / \sqrt{1-v^{2} c^{-2}}$ [2], of the mechanical body including its variable heat-energy $Q\left(v^{2}\right)=$ $E_{o} \sqrt{1-v^{2} c^{-2}}$. The relativistic transport of the body's internal heat obeys the Einstein-Laue relativistic law, $Q\left(v^{2}\right)=Q_{o} \sqrt{1-v^{2} c^{-2}}$. Therefore, the internal rest-energy $E_{o}=M_{o} c^{2}$ can be considered as the relativistic heat energy of internal kinematic chaos, $Q_{o} \equiv E_{o}$. The Einstein's mechanical body loses its internal heat-energy under the spatial motion like the quantum particle in wave mechanics loses its energy-frequency, $\hbar \omega=\hbar \omega_{o} \sqrt{1-v^{2} c^{-2}}$. Any Einstein-Laue law is, in fact, the relativistic law of proper time dilation in the Mikowski spacetime, which is not inherent in Newtonian mechanics.

\footnotetext{
ae-mail: bulyzhenkov.ie@mipt.ru
} 
From the point of view of the observer who calculates the energy balance according to the laws of Einstein's relativistic theory, an inertial body with rest energy $E_{o}=M_{o} c^{2}$ loses some of its internal energy both at high speeds and at small ones, when $E_{o} \sqrt{1-v^{2} c^{-2}}-E_{o} \approx-M_{o} v^{2} / 2$.

Recall from the Lorentz transformations for a rest-energy $E_{o}=\gamma(E-v P)$ at zero net momentum $P_{o}=\gamma\left(P-v c^{-2} E\right)=0$ that the full kinetic energy $E=E_{o} / \sqrt{1-\beta^{2}}$ of a moving body,

$$
E=E_{o} \sqrt{1-\beta^{2}}+v P \equiv E_{o} \sqrt{1-\beta^{2}}+\beta^{2} E \equiv E_{o} \sqrt{1-\beta^{2}}+\frac{E_{o} \beta^{2}}{\sqrt{1-\beta^{2}}}
$$

contains the variable heat-energy $Q\left(v^{2}\right) \equiv E_{o} \sqrt{1-\beta^{2}}$ of internal kinematic chaos and the variable kinetic energy of ordered translations $T\left(v^{2}\right)=\beta^{2} E_{o} / \sqrt{1-\beta^{2}}$. These variable items form the Hamilton function $H=-L+v \cdot P$, which lies in the foundation of quantum mechanics. Once one accepts the variable heat transport instead of the constant mass transport, then relativistic mechanics with the internal degree of freedom converges to quantum mechanics for similar transport of energy. The counterflow of heat losses, $\left(-M_{o} v^{2} / 2\right)$ at $v^{2} \ll c^{2}$, halves the kinetic energy flow $E_{o} \beta^{2}$ under spatial translations of the thermodynamic body, because $\left(M_{o} v^{2}-M_{o} v^{2} / 2\right)=M_{o} v^{2} / 2$. Once again, the kinetic energy $M_{o} v^{2} / 2$ in the Newton model does not coincide with the real energy of slow translations $M_{o} v^{2}$ in the Einstein relativistic physics and in the Hamilton / Lorents formalism for relativistic physics and quantum mechanics. Thus, it is not possible to consider Newton's model dynamics with the halved kinetic energy of cold masses as the correct physics theory "in the field of its applicability". And there is no much sense in Newtonian nonrelativistic referents for the relativistic theory with new (internal) degree of energy freedom.

\section{Relativistic energy-charges with internal heat}

The Newton mechanics maintains transport and conservation of the constant mass, as well as transport and conservation of its kinetic energy $M_{o} v^{2} / 2$ next to the potential one. Relativistic physics has only one conservation law for the united mass-energy and describes only transport of energy, including internal heat. The Newton concepts of cold constant masses is a model for low speed flows of energy. In reality, the non-Newtonian time dilation is well tested due to the many-kilometer motion of shortliving muons in the Earth atmosphere. Because of the more comprehensive notion of the internal heat-energy $Q\left(v^{2}\right)=M_{o} c^{2} \sqrt{1-\beta^{2}}$ one must completely abandon the classical mass notion $M_{o}=$ const for a real thermodynamical body or for an indivisible particle. The mechanical inertia is a purely energy characteristic since it grows with absorption of " massless electromagnetic waves". The unnecessarily preservation of two competing notions (energy vs mass) for the inertial charge in mechanics inevitably leads at first to physical confusions, and then to incorrect mathematical models. Below we demonstrate the inability of the Newtonian theory of cold masses to act as a particular limit for Einstein's physics of inertial energies with variable internal heat.

The probe motion of any small body with the rest-frame heat-energy $E_{o}$ is described in any metric gravitational field $g_{o o}$ by the full relativistic energy[2] $E=E_{o} \sqrt{g_{o o}} / \sqrt{1-\beta^{2}}$, which we rewrite as

$$
E \equiv q \varphi_{o}=E_{o} \sqrt{1-\beta^{2}}+\frac{E_{o} \beta^{2}}{\sqrt{1-\beta^{2}}}-\frac{E_{o}\left(1-\sqrt{g_{o o}}\right)}{\sqrt{1-\beta^{2}}} \equiv Q+T+U .
$$

This full energy is proportional to the value of the total inertial (and gravitational) charge $q \equiv E / \varphi_{o}$, where $\varphi_{o} \equiv c^{2} / \sqrt{G}=1.04 \times 10^{27} \mathrm{eB}$. In the absence of ordered rotations, this charge gains contributions from the internal heat-energy, $Q \equiv E_{o} \sqrt{1-\beta^{2}}>0$, of the relativistic body, from its kinetic 
energy of spatial translations as a whole system, $T \equiv E_{o} v^{2} / c^{2} \sqrt{1-\beta^{2}}>0$, and from the negative potential energy in an external gravitational field, $U \equiv-E_{o}\left(1-\sqrt{g_{o o}}\right) / \sqrt{1-\beta^{2}}<0$, where $0<g_{o o} \leq 1$ and $\beta^{2} \equiv v^{2} / c^{2}<1$. Ernst Mach would have approved, probably, our definition of the energy-type charge $q$, because its inertia and gravitation depends on the spatial distribution of all other charges through gravitational energy contributions $U$.

The energy-type charge $q$ with the heat part $Q / \varphi_{o}$ is constant only in constant external fields, when $\partial_{t} g_{o o}=0$. The positive kinetic energies of the internal (chaotic) and external (ordered) motions can be summered, $K \equiv Q+T=E_{o} / \sqrt{1-\beta^{2}}$, to trace their net increase in the beginning of the free gravitational fall. This sum was implemented in Special Relativity (SR) exclusively in favor of old referents of Newtonian dynamics with degenerated kinematic degrees of freedom. In other words, SR was oriented in its nonrelativistic limit on the Newtonian kinetic term $E_{o} \beta^{2} / 2$ for the mixed transport of constant masses and bound internal + external energies. Contrary to its capabilities, SR did not reveal internal energy changes within moving bodies. The point is that rapid changes of the intrinsic heat-energy $E_{o} \sqrt{1-\beta^{2}}$ take place in small probe bodies under collinear counter currents of thermal (chaotic) and translation (ordered) energies. The model dynamics of Newton's cold masses should in no way serve as a non-relativistic referent for a continuous medium of inertial flows with a noncollinear transport of disordered (heat) and ordered (regarding to an observer) energies. Instead of the vector transport of two variable energies, Newtonian mechanics operates with the unphysical transport of constant masses. Numeral algebra for vector flows of heat and translation energies can work only for collinear exchange mechanisms, but not for independent dissipation directions in $3 \mathrm{~d}$ space.

The relevance of Newton's theory should be canceled not only for the SR slow dynamics of inertial energies with independent heat exchanges, but also for the search of metric solutions of General Relativity (GR) through the Newtonian limit of a weak field. Indeed, the inhomogeneous metric component $g_{o o}(x)$ can be uniquely expressed from the relativistic balance (2) through the universal ratio $U / E \leq 0$ of the negative gravitational contribution $U$ to the full energy content $E$, including internal heat $\mathrm{Q}$ :

$$
\sqrt{g_{o o}} \equiv(Q+T+U) \frac{\sqrt{1-\beta^{2}}}{E_{o}} \equiv 1+\frac{U \sqrt{g_{o o}}}{E} \equiv \frac{1}{[1+(-U / E)]} .
$$

On the basis of algebraic identities in relations (2) - (3), one can formulate the following $g_{o o}$ - theorem: " The purely temporal metric component is defined in the gravitation of Mach-Einstein energies by a continuous gravitational contribution $\varphi \equiv U / E$ strictly as $g_{o o}=(1-\varphi)^{-2}$, and without singularities on the entire half-interval $-\infty<\varphi \leq 0$ of admissible arguments".

The radial function $\varphi=-G E_{S \text { un }} / c^{4} r$ in strong fields corresponds to the singularity free metric component $g_{o o}(r)=\left[c^{4} r /\left(c^{4} r+G E_{S u n}\right)\right]^{2}$. Therefore, the Schwarzschild metric [3] with $g_{o o}(r)=$ $\left(c^{2} r-2 G M_{S \text { un }}\right) / c^{2} r$ does not fully agree with the Mach-Einstein gravity [4] of total energies with variable internal heat. It is simply unjustifiable to equate the model gravitation of Newton's cold and constant masses to the Einstein gravitation of energy-charges with internal heat. In 1938, Einstein criticized the duality of massive particles and massless fields, pointing to the non-dual way in the relativistic physics of continuous carriers of energy [5]. After that, the author of General Relativity firmly characterized the Schwarzschild 4-interval with a singularity, which "is not related to physical reality" [6].

Recall that in 1913 Einstein and Grossmann introduced a gravitational field only into the time part of the Minkowski space-time interval. They also preserved, at that time without variants, the point mass model or localized substance for comparison of relativistic and classical motions in gravitational fields [7]. After correspondence with Gilbert, Einstein had a tensor equation for gravitational fields. The space-time interval $d s=\sqrt{d \tau^{2}-d l^{2}}$ became a combination of two warped sub-intervals, $d \tau^{2} \equiv$ 
$g_{o o}\left[d t+\left(g_{o i} d x^{i} / g_{o o}\right)\right]^{2}$ and $d l^{2} \equiv\left(g_{o i} g_{o j} g_{o o}^{-1 / 2}-g_{i j}\right) d x^{i} d x^{j}$. In this case, the non-geometrized substance in the right side of the Einstein Equation [8] again allowed spatial localization and empty space regions in accordance with Newton's referential presentations of localized inertial masses. But where is the next revolution of new Einstein's ideas of 1938-39 after the first shakes of the foundation of gravity in 1913-1916?

\section{Deceleration by strong field and accelerated takeoff from the center}

The Schwarzschild metric for curved emptiness was rejected for physical reality not only by Einstein. A curved 3-space in the metric decision of 1916 for a constant central field [3] could not suit many physicists. Sommerfeld, Schwinger, Feynman and many others sought to preserve the Euclidean nature of electrodynamics, including its constant Gaussian flow along a two-dimensional closed surface and the strict Bohr-Sommerfeld quantization rule over a one-dimensional closed contour. In the 80s of the last century, academician A. Logunov suggested the alternative theory of gravitation [9] by returning back to the Minkowski space-time of 1908. This would might return flat space to relativistic physics, while retaining the referents of Newtonian dynamics in " areas of joint applicability of theories". And it would might eliminate nonphysical black holes in strong fields, and would might lead to a cyclical state of matter with high and low density.

Logunov's program goals could be welcomed, especially since Einstein himself tried to improve the relativistic theory. However, Einstein understood that consistent classical mechanics should be constructed, like the quantum mechanics of the distribute particle, in non-dual terms of extended energy densities: "The substance - where the energy concentration is large, the field - where the energy concentration is small. This difference is not qualitative, but rather quantitative. There is no point in treating matter and the field as two qualities, completely different from each other. We can not imagine a definite surface that clearly divides the field and matter. '

The non-dual theory of energy densities can not be transformed into a dual one because of the transition from high concentrations of matter to low. It is unreasonable to expect that non-dual objects in physics of the microworld will begin to change into dual ones when the scale of space or the speed of particle motion changes. The model separation of the unified energy essence (in the macrocosm of a continuous material space) into an allegedly localized substance and the noninertial field is explained by the quantitative principles of observing nonlocal reality. The observations can not be justified as true through the mathematically right theory like Newton's incomplete dynamics for cold massive points. In 1938, Einstein proposed not to return back to the sum of three concepts (space-time plus field plus matter, as Logunov chose [9]) instead of the two concepts of 1916 (space-time-field plus substance). On the contrary, he proposed to go ahead and to reduce the number of physical concepts to one, introducing the continuous density of the extended elementary particle into a united space-timefield-substance. This revolutionary proposal was not accompanied at that time by new formulas and verifiable predictions. But Einstein immediately warned that in the non-dual theory of a pure field, a continuous particle must disappear from the auxiliary right-hand side of the 1916 Equation. Today everyone can check in support of the non-dual ideas of Einstein that the self-contained metric of GR on the energy balance (2) with the theorem (3) for $g_{o o}(r)=r^{2} /\left(r+r_{o}\right)^{2}$ allows us to get the flat spatial section $d l^{2}=\delta_{i j} d x^{i} d x^{j}$ in the vanishing Einstein curvature [4], $G_{o o}=0$ or $R_{o o}=g_{o o} R / 2 \neq 0$.

To predict new testable effects in the Descartes paradigm of the nonempty space of extended mass-energies 1938, it is necessary to reject Newtonian referents of the point mass in the vacuum and to trace in (2)-(3) the dynamical energy balance of warmed bodies with the Einstein-Laue heat transport. It is sufficient to make the assumption that the relativistic rest energy $E_{o}$ has a kinetic nature due to chaotic motion of inertial densities of a probe body or an elementary particle. The remote 
probe body at rest will have in (3) a maximum of its internal kinetic energy $Q(\infty)=E_{o}$ under zero mechanical energy: $M(\infty) \equiv T(\infty)+U(\infty)=0$ for $T(\infty)=0, U(\infty)=0$. At any final removal of $R$ from the static gravitational center, the probe body at rest, $\beta(R)=0$ and $T(R)=0$, continues to possess the maximum internal (kinetic) heat-energy $Q(R)=E_{o}$ under a negative mechanical energy $M(R)=U(R)=-E_{o}\left[1-\sqrt{g_{o o}(R)}\right]<0$. In this case, the total energy of the probe body, $E(R)=$ $Q(R)+M(R)=E_{o} \sqrt{g_{o o}(R)}>0$, always stays in (3) positive.

If a probe body at rest is released at any distance $R$ from the center of strong gravity, then this body will tend to its kinetic equilibrium due to the theorem on equipartition of kinetic energies over available degrees of freedom,

$$
Q\left(r_{e q}\right) \equiv E_{o} \sqrt{1-\beta^{2}\left(r_{e q}\right)}=\frac{E_{o} \beta^{2}\left(r_{e q}\right)}{\sqrt{1-\beta^{2}\left(r_{e q}\right)}} \equiv T\left(r_{e q}\right),
$$

and will reach $v^{2}\left(r_{e q}\right)=c^{2} / 2$ (in the absence of rotations) at the equilibrium distance $r_{e q}<R$.

The reason of mutual gravitation of both interacting partners is the universal tendency to equipartition of chaotic (internal) and ordered (translation) kinetic energies. Without the emergence of gravitational forces, the inertial state would not change. Indeed, the rate of kinetic energy $T$ changes can not be compensated in (4) only by the rate of internal energy $Q$ changes under accelerations/decelerations. For the spontaneous growth of translational speed and the striving for an equilibrium distribution of internal and translation kinetic energies, inertial bodies mutually create potential gradients (and conditions for mutual rotations). Again, the essence of the paired forces of gravity is to cater for mutual aspirations to equal distribution of kinetic energy over available degrees of freedom, including internal chaotic motions, reciprocal translations and counter-rotating motions.

Let us return to the vertical drop of a particle from an initial height $R$ to a static center with high energy $E_{S \text { un }} \gg E_{o}$. The full energy $E(R)=E_{o} \sqrt{g_{o o}(R)}$ of a falling particle in the static gravitational field $g_{o o}(r)$ is constant in General Relativity, where $E[r(t)] / E_{o}=\sqrt{g_{o o}(r) /\left[1-\beta^{2}(r)\right]}=\sqrt{g_{o o}(R)}$. Such conservation allows to associate with the metric field $g_{o o}(r)$ the physical speed, $0 \leq v^{2}(r) \equiv$ $\left(d r / \sqrt{g_{o o}} d t\right)^{2}<c^{2}$, which monotonously increases under a vertical fall from zero to the speed of light. However, a remote observer can fix coordinate displacements $d r$ of probe bodies only with respect to the local time, which is $d t \equiv d x^{o} / c$ in the absence of gravitation. Therefore, all remote observations are related to dynamics of the coordinate velocity $d r / d t$ in the following relations:

$$
\frac{g_{o o}(r)}{g_{o o}(R)}=1-\frac{v^{2}(r)}{c^{2}} \equiv 1-\frac{1}{g_{o o}(r) c^{2}}\left(\frac{d r}{d t}\right)^{2} .
$$

Taking into account two opposite directions of radial motions, the coordinate velocity in (5) can be rewritten in an equivalent form with the introduction of a unit vector $\hat{\mathbf{r}} \equiv \mathbf{r} / r$ :

$$
\frac{d \mathbf{r}(t)}{d t}= \pm \hat{\mathbf{r}} c \sqrt{g_{o o}(r)\left[1-\frac{g_{o o}(r)}{g_{o o}(R)}\right]} .
$$

It turns out from (6) that the observed body first dials the coordinate speed, $\mathbf{0} \rightarrow d \mathbf{r} / d t \rightarrow$ $-\hat{\mathbf{r}} c \sqrt{g_{o o}(R)} / 2$, in the region of the moderate fields $g_{o o}(R) \geq g_{o o}\left(r \rightarrow r_{e q}\right) \geq g_{o o}(R) / 2$. Then, with further growth of the field as the probe body moves toward the center in the region $g_{o o}(R) / 2 \geq g_{o o}(r \rightarrow$ $0) / \geq 0$, this body begins to decelerate to zero coordinate speed, $-\hat{\mathbf{r}} c \sqrt{g_{\text {oo }}(R)} / 2 \rightarrow d \mathbf{r} / d t \rightarrow \mathbf{0}$. In this case, the magnitude of the physical speed $|\mathbf{v}|=\left|d \mathbf{r} / \sqrt{g_{o o}(r)} d t\right|$ monotonically increases from zero to the speed of light on the whole trajectory of the free fall to the center of the radial gravitational field. The body completely loses its internal heat-energy $Q(0)=0$ at the end of the fall. The lack of this 
rest-frame energy allows the physical velocity to reach formally the speed of light in the very center of gravity. In contrast to the physical speed $v \rightarrow c$, the coordinate velocity is zeroed at the center, $d r / d t \rightarrow 0$, where the body temporarily stops before the reverse takeoff from the center.

After a nonequilibrium stop in the extreme position with $Q(0)=0, M(0)=T(0)+U(0)=$ $E_{o} \sqrt{g_{o o}(R)}$ in the center of the radial gravitational field, the test body starts its reverse accelerated motion to the equilibrium point of the kinetic energies $Q\left(r_{e q}\right)=T\left(r_{e q}\right)=E_{o} \sqrt{2} / 2$ for $v^{2}=d r^{2} / g_{o o} d t^{2}=c^{2} / 2$. Here the maximum of observed (coordinate) speed $d r / d t=c \sqrt{g_{o o}(R)} / 2$ will be again achieved at $g_{o o}\left(r_{e q}\right)=g_{o o}(R) / 2$. After passing through the equilibrium distance from the center, $r(t)=r_{e q}$, the probe body will continue to increase its internal energy $Q(r)$ and returns with the weak field deceleration of Newton to the height $R$. This initial state will have again $E=E_{o} \sqrt{g_{o o}(R)}$, $Q(R)=E_{o}, T(R)=0$, and $U(R) / E_{o} \sqrt{g_{o o}(R)}=1-g_{o o}^{-1 / 2}(R)<0$.

In order to find the observed (coordinate) acceleration of a probe body on the entire trajectory of the free fall and the reverse takeoff from the field center, it is sufficient to differentiate the coordinate velocity (6) over the world (coordinate) time $t$. The computation rule $d g_{o o}(r[t]) / d t=\left(d g_{o o} / d r\right) d r / d t$ can yield the strong field acceleration/deceleration law:

$$
\frac{d^{2} \mathbf{r}(t)}{d t^{2}}=\hat{\mathbf{r}} c^{2}\left(\frac{1}{2}-\frac{g_{o o}(r)}{g_{00}(R)}\right) \frac{d g_{o o}(r)}{d r} \Rightarrow\left\{\begin{array}{l}
-\left(1-\frac{4 r_{o}}{r}\right) \frac{c^{2} r_{o} \mathbf{r}}{r^{3}}, g_{o o}^{1916}=1-\frac{2 r_{o}}{r} \\
-\frac{c^{2} r_{o} \mathbf{r}\left(r^{2}-2 r_{o} r-r_{o}^{2}\right)}{\left(r+r_{o}\right)^{5}}, g_{o o}^{2008}=\frac{r^{2}}{\left(r+r_{o}\right)^{2}}
\end{array}\right.
$$

If a body falls to the center from large distances, $R / r_{o} \Rightarrow \infty$ and $g_{o o}(\infty)=1$, then the metric component $g_{o o}\left(r_{e q}\right)=1 / 2$ gains a half of its original value under the equilibrium of kinetic energies. This result is independent from particulars of competing gravitational theories. For a formal comparison of the predictions of the equipartition theorem in application to different metric solutions, we used at the right hand side of (7) both the Schwarzschild metric of 1916 for the empty space around a point source and the nonempty space metric solution [4] $g_{o o}^{2008}=r^{2} /\left(r+r_{o}\right)^{2}$ from the algebraic identity (3) with $U / E=-r_{o} / r$, where $r_{o} \equiv G E_{2} / c^{4}$. In both cases, the Newtonian acceleration by the weak field $-c^{2} r_{o} \mathbf{r} / r^{3}$ smoothly changes the sign for the deceleration at $r>2 r_{o}$, i.e. above the supposed black hole horizon in dual physics with empty space.

\section{Conclusions on verifiable predictions}

The gravitational theory of 1916 with the Newtonian referents of the metric predicts the presence of a gravitational horizon at the distance $r=2 G E_{2} / c^{4} \equiv 2 r_{o}$ from the center of the gravitating energy $E_{S \text { un }}=r_{o} \varphi_{o}^{2}$. The metric solution of General Relativity in the 1938 pure field paradigm of the nonempty space of radial mass-energies predicts a smooth decrease of $g_{o o}(r)=r^{2} /\left(r+r_{o}\right)^{2}$ and the observed motion of probe bodies up to $r=0$. Observational confirmation or refutation of the horizon of events in the galaxy center can falsify or verify, respectively, the 1938 proposal of Einstein and Infeld regarding transition of General Relativity to the non-dual field way for analytical description of matter. The identity of substance and strong fields supports the double unification criterion: to unite particles with fields and electricity with gravitation [10].

Coordinate deceleration of falling matter in extreme fields and the accelerated takeoff from the gravitational center in (7), as if along a degenerate Keplerian orbit, could, upon detection, clarify the nature of gravity through the equipartition theorem for kinetic energies on internal and external degrees of freedom. In this direction, it is advisable to simulate the Metagalaxy expansion with acceleration basing on the previous cycle of the Metagalaxy compression with deceleration. 
So far we neglected radiative exchanges of a moving carrier of internal relativistic heat with third bodies or with surrounding matter, which plays the role of a world thermostat. In practice, an increase of internal energy and temperature under the physical speed decrease relatively to the world thermostat is to be accompanied by an increase in radiation according to the Stefan-Boltzmann law. Therefore, the explanation of gravity through the theorem on the equipartition of internal and translation kinetic energies can be tested by non-Newtonian self-warming of small cosmic bodies, like decelerated asteroids or comets. The variable kinematic heat predicts temperature changes up to volcanic activities of rapidly decelerated bodies, like satellites of large planets, under sharp changes of their velocities regarding the world thermostat or the cosmic microwave background.

The fall to the center of the galaxy from its periphery is characterized by the cooling of thermodynamic bodies and by the nonequilibrium absorption of external radiation. Such absorption imitates dark regions without matter for IR and optical observations. The non-geodesic deceleration of matter in inelastic collisions near the center of the galaxy (as well as near each massive center) leads to its local heating and to the launch of a thermonuclear reaction for the synthesis of light elements. The thermonuclear energy in the center of a star heats its surface due to thermal conductivity that results in visible luminosity of moving stars even at the edge of the galaxy. Here other accelerated bodies are cooling according to the Einstein-Laue law for the relativistic heat transport.

The stars, which are on elongated elliptical orbits around the massive center, are warmed twice per cycle because of deceleration by small circular satellites at minimum approximations of the star to this center. In this case, the rapid loss of translational kinetic energy occurs according to the EinsteinLaue law with the explosive growth of internal heat-energy. Thus, the gravitation until equipartition of kinetic energies predicts a double burst of luminosity of stars at each cycle along an elongated orbit. Upon detection, such thermokinetic effects can be modeled for a numerical verification of changes in internal energy according to the relativistic heat transport.

The relativistic law of releasing the internal kinetic energy of mechanical bodies or liquids is important to take into account quantitatively even with small changes in their velocity in the laboratory. Contrary to the collinear transport of internal and translation energies in small probe bodies, heat transfer in media can be directed not along the mechanical momentum of moving particles. This vector imbalance can be interpreted through non-Newtonian additional contributions to the mechanical energy flows in nonrelativistic hydro and gas dynamics, despite the full energy conservation of the moving medium. In general, Newton-Euler dynamics of ideal liquids can not be extended on Navier-Stokes dissipative flows without Laue heat losses under motion.

\section{References}

[1] T.S. Kuhn, The Structure of Scientific Revolutions (Chicago, 1962)

[2] L.D. Landau and E.M. Lifshitz, The Classical Theory of Fields, Fourth Edition: Volume 2 (Course of Theoretical Physics Series) 4th Edition (Butterworth-Heinemann, 1980)

[3] K. Schwarzschild, Sitzungsber. Deut. Akad. Wiss., Berlin, 189 (1916)

[4] I.E. Bulyzhenkov, Int. Jour. Theor. Phys. 47, 1261 (2008)

[5] A. Einstein, L. Infeld, The Evolution of Physics (Cambridge Press, 1938)

[6] A. Einstein, Annals of Mathematics 40, 922 (1939)

[7] A. Einstein and M. Grossmann, Math. und Phys. 62, 225 (1915)

[8] A. Einstein, Annalen der Physik 49, 769 (1916)

[9] A.A. Logunov, The Theory of Gravity (Nauka, Moscow, 2001)

[10] I.E. Bulyzhenkov, Bulletin Lebedev Phys. Inst. 43, 138 (2016) 